\title{
Improving lipid management in patients with acute coronary syndrome: The ACS Lipid EuroPath tool
}

\author{
Alessandro Sionis ${ }^{\mathrm{a},}{ }^{*}$, Alberico L. Catapano ${ }^{\text {b, }}{ }^{* *}$, Gaetano M. De Ferrari ${ }^{\mathrm{c}}$, Dariusz Dudek ${ }^{\mathrm{d}}$, \\ J. Wouter Jukema ${ }^{\mathrm{e}}$, Ulf Landmesser ${ }^{\mathrm{f}}$, Angela Pirillo ${ }^{\mathrm{g}}$, François Schiele ${ }^{\mathrm{h}}$, Azfar Zaman ${ }^{\mathrm{i}}$, \\ Jose L. Zamorano ${ }^{\mathrm{j}}$ \\ ${ }^{a}$ Intensive Cardiac Care Unit, Cardiology Department, Hospital de La Santa Creu I Sant Pau, Biomedical Research Institute IIB-SantPau, Universidad \\ Autonoma de Barcelona, Barcelona, And Centro de Investigación Biomédica en Red Enfermedades Cardiovasculares (CIBERCV), Instituto Carlos III, Madrid, \\ Spain \\ b Department of Pharmacological and Biomolecular Sciences, University of Milan, Milan, IRCCS MultiMedica, Sesto S. Giovanni, Milan, Italy \\ ${ }^{\mathrm{c}}$ Department of Medical Sciences, Division of Cardiology, Azienda Ospedaliero Universitaria Città Della Salute e Della Scienza di Torino, Torino, Italy \\ d Jagiellonian University Hospital, Krakow, Poland \\ e Department of Cardiology, Leiden University Medical Center, Leiden, And Netherlands Heart Institute, Utrecht, the Netherlands \\ ${ }^{\mathrm{f}}$ Department of Cardiology, Charité University Medicine Berlin, German Center for Cardiovascular Research (DZHK), Partner Site Berlin, Berlin Institute of \\ Health (BIH), Berlin, Germany \\ ${ }^{g}$ Center for the Study of Atherosclerosis, E. Bassini Hospital, Cinisello Balsamo, Milan, and IRCCS MultiMedica, Sesto S. Giovanni, Milan, Italy \\ ${ }^{\mathrm{h}} \mathrm{CHU}$ of Besançon, Cardiology Department, Besançon, France \\ ${ }^{i}$ Newcastle Upon Tyne Hospital NHS Foundation Trust, Freeman Hospital, And Newcastle University, Newcastle Upon Tyne, UK

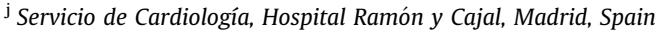

\section{Keywords:}

Acute coronary syndrome

Dyslipidemia

Lipids

Guidelines

Self-assessment

\begin{abstract}
A B S T R A C T
Post-acute coronary syndrome (ACS) patients are at very high risk for recurrent events and mortality, despite the availability of effective pharmacological approaches. In 2018, the ACS EuroPath Survey, performed in collaboration with 555 European cardiologists, identified a sub-optimal LDL-C management in post-ACS patients.

Based on these premises, the ACS EuroPath II project led to the development of a self-assessment tool to improve lipid management in these very high risk patients, taking into consideration the new 2019 ESC/EAS guidelines. This tool is built in 3 sections. The first is a questionnaire to assess the lipid management practice from the acute phase up to 12 months of follow-up. The main topics covered in this section relate to 1 ) acute phase (lipid management of ACS patients during hospitalization; 2 ) discharge (lipid management at discharge, with focus on follow-up plan); 3) follow-up (lipid management at the time of first and subsequent follow-ups); 4) referral pathway for definitive lipid management care of post-ACS patients; 5) evaluation of the achieved goal at 6 months to 1 year and key implications. The second section is a brief report to position the results against other European Union clinical practice and European guidelines. The last section allows the physician to evaluate and consider the implementation of one or more strategies, successfully developed in leading European centers, in order to optimize their own clinical practice.
\end{abstract}

๑) 2021 The Authors. Published by Elsevier B.V. This is an open access article under the CC BY-NC-ND license (http://creativecommons.org/licenses/by-nc-nd/4.0/).

\footnotetext{
* Corresponding author. Intensive Cardiac Care Unit, Cardiology Department, Hospital de la Santa Creu i Sant Pau, Biomedical Research Institute IIB-SantPaul, Universidad Autonoma de Barcelona, Barcelona, Spain.

** Corresponding author. Department of Pharmacological and Biomolecular Sciences, University of Milan and IRCCS Multimedica Via Balzaretti, 9 - 20133, Milan, Italy.

E-mail addresses: asionis@santpau.cat (A. Sionis), alberico.catapano@unimi.it (A.L. Catapano).
}

\section{Introduction}

Acute coronary syndrome (ACS) patients remain at very high risk for recurrent events and mortality, despite availability of effective pharmacological approaches [1,2]. In 2018, the ACS EuroPath I survey, performed in collaboration with 555 European cardiologists and 2775 patient records, demonstrated a sub-optimal LDL-C management after ACS [3]. However, multiple initiatives 


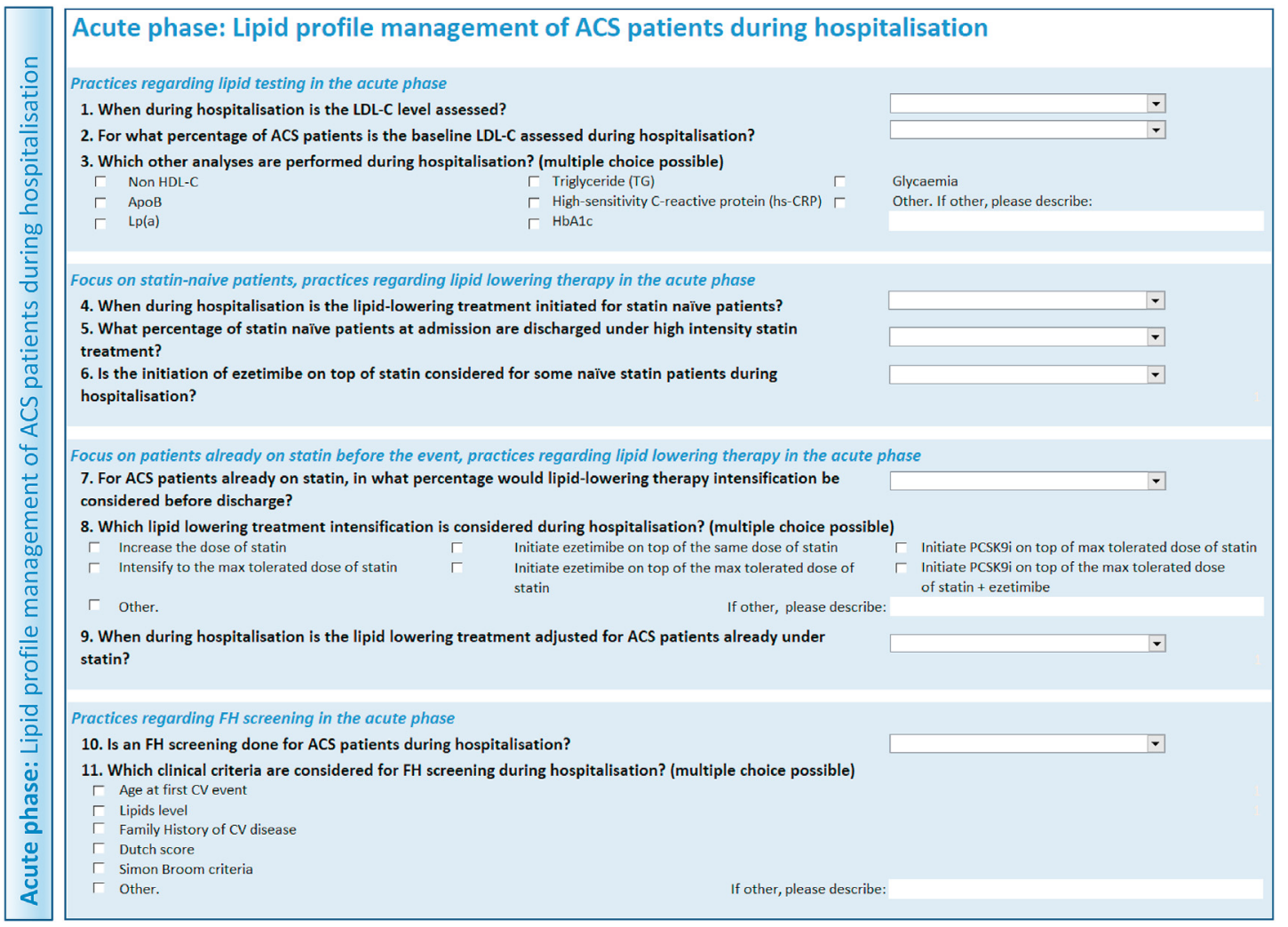

Fig. 1. Lipid profile management of ACS patients during the acute phase (hospitalization).

from several European countries have shown that the implementation of robust algorithms/protocols for LDL-C management can result in a more effective translation of guideline recommendations into clinical practice and significantly increase the achievement of recommended LDL-C goals to reduce CV risk [4]. Based on these premises, the ACS EuroPath project led to the development of a self-assessment tool for physicians aimed at identifying potential areas of improvement in the management of these very high risk patients, taking into consideration the recommendations provided by the new 2019 ESC/EAS guidelines [5]. This self-assessment tool has been developed in collaboration with a team of 8 European cardiologists and lipidologists and is presented in this article.

\section{Objectives of the self-assessment tool}

The objectives of the self-assessment tool are to: 1) provide a snapshot of clinical practice at a given time (it does not require identifiable patient related data). It is based on simple questions to assess personal practice regarding post ACS lipid management. The tool is quick to use and data are only for personal use of the physician, 2) position the results with regard to current European clinical practice as highlighted by the 2018 ACS EuroPath I survey [3], and 3) help identify strengths and areas of optimization by comparing to current European guidelines and European local clinical practice.

\section{Key topics covered by the self-assessment tool}

The key topics covered in the self-assessment tool relate to the management of lipids in ACS patients from the hospitalization phase and during follow-up and has been organized in 3 sections: a questionnaire, a report, and potential targets for optimization.

\section{Questionnaire}

The questionnaire allows the physician to insert patient information from acute admission to the follow-up phase and comprises four distinct sections: 1) acute phase (lipid management of ACS patients during hospitalization; 2) discharge (lipid management at discharge, with focus on follow-up plan); 3) follow-up (lipid management at the time of first and subsequent follow-ups); 4) referral pathway for lipid management of post-ACS patients; 5) evaluation of the achieved goal at 6 months to 1 year and key implications.

The acute phase section includes practices regarding lipid testing, lipid lowering therapy (with distinction between statinnaïve patients and patients already on statin at admission), and practices regarding the screening for heterozygous familial hypercholesterolemia (FH). Physicians must indicate when the LDL-C level is assessed, for what percentage of ACS patient LDL-C level is assessed at admission, and additional parameters evaluated (including total cholesterol, non-HDL-C, Lp(a), and glycaemia among others) during the acute phase (Fig. 1). Information regarding lipid-lowering therapy at admission is collected. For statin naïve patients, physicians must indicate when the lipidlowering treatment is started after admission, the percentage of patients receiving high intensity statin post-event, and whether ezetimibe on top of statin is considered in specific subgroup of patients. For patients already on statin before the event, physicians should indicate the percentage of patients who had a lipidlowering therapy intensification, the type of treatment intensification (dose increase, dose doubling, adding ezetimibe on top of maximally tolerated dose of statin, initiate PCSK9i, etc.), and when 


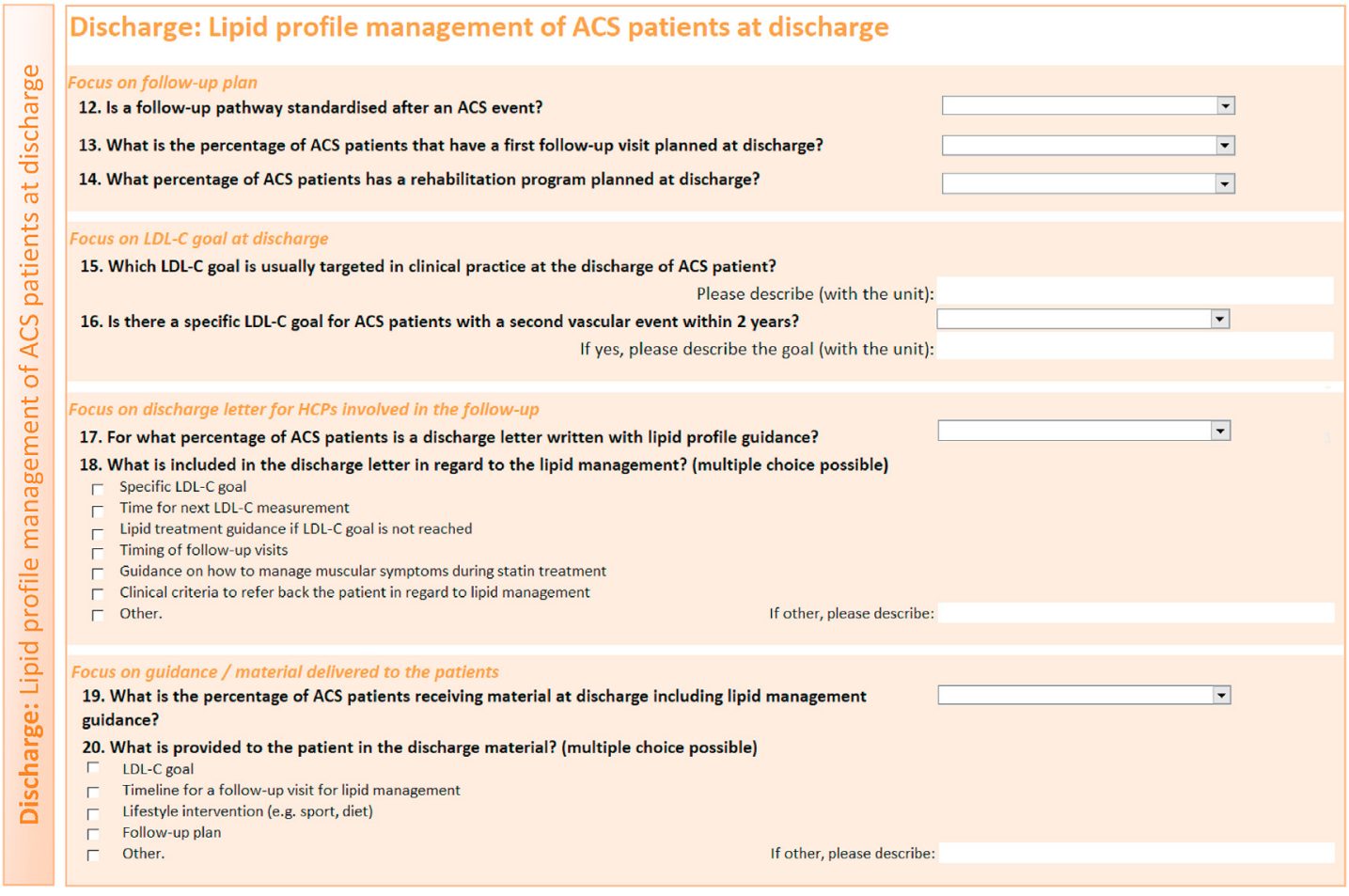

Fig. 2. Lipid profile management of ACS patients at discharge.

the treatment is adjusted with regard to admission time (Fig. 1). A special section in the questionnaire is dedicated to the detection of patients with $\mathrm{FH}$. Based on recommendations by ESC/EAS guidelines and the data provided by surveys in ACS patients (refs), physicians are asked to provide information on whether a screening protocol is applied in patients hospitalized for an ACS, and the clinical criteria considered for the screening (in particular age, family history of CVD, elevated lipid levels, and the clinical score used) (Fig. 1). This represents a significant point since, although several surveys have reported an increased incidence of $\mathrm{FH}$ among
ACS patients compared with the general population, the EuroPath I survey showed that only $18 \%$ of patients hospitalized for an ACS were screened for FH during the acute phase (Landmesser et al.) [3].

The discharge section focuses on four specific points: 1 ) followup plan, 2) LDL-C goal at discharge, 3) discharge letter addressed to the general practitioner (GP) involved in this phase, and 4) information provided to patients (Fig. 2). ESC/EAS guidelines recommend that ACS patients participate in a structured cardiac rehabilitation program, in order to improve adequate control of cardiovascular risk factors including lipid levels and improve

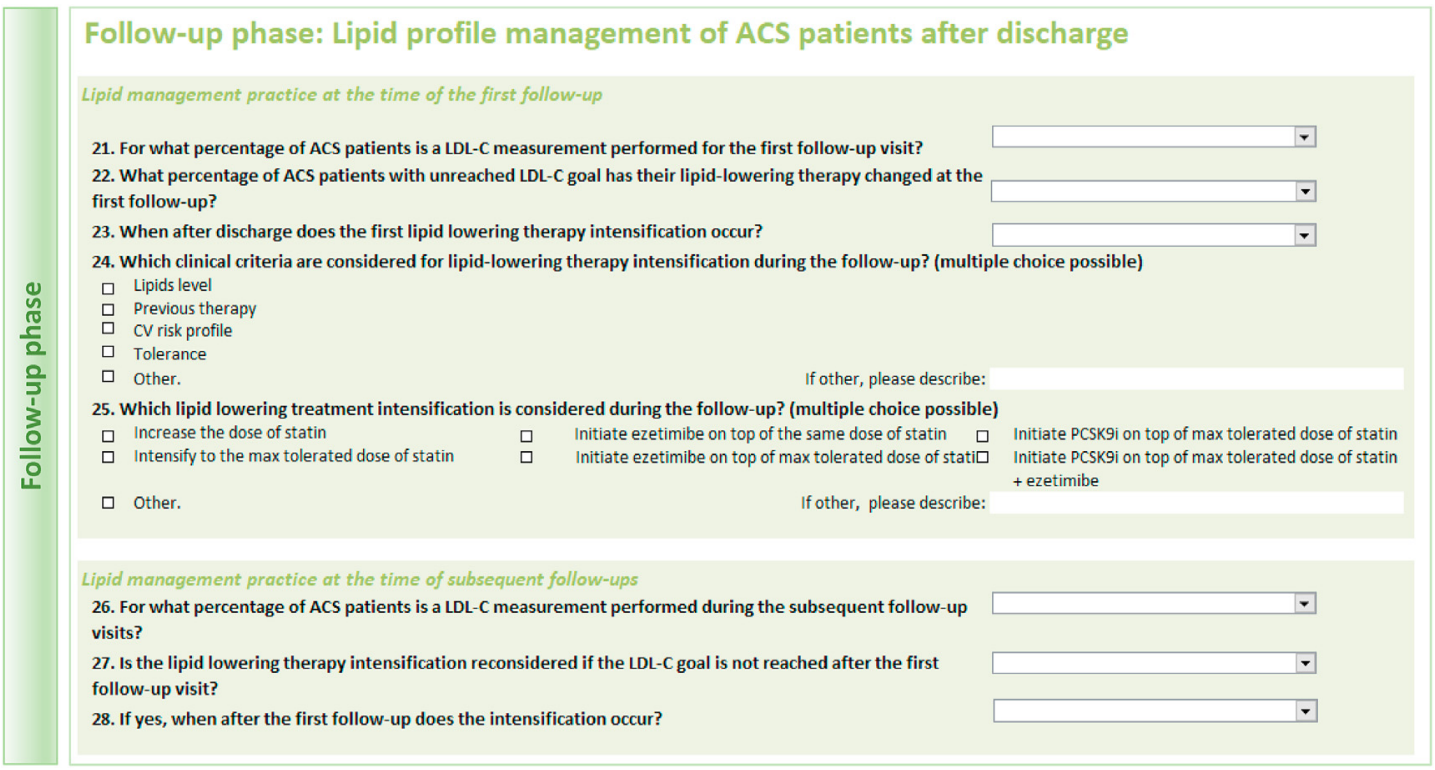

Fig. 3. Lipid profile management of ACS patients after discharge (follow-up phase). 


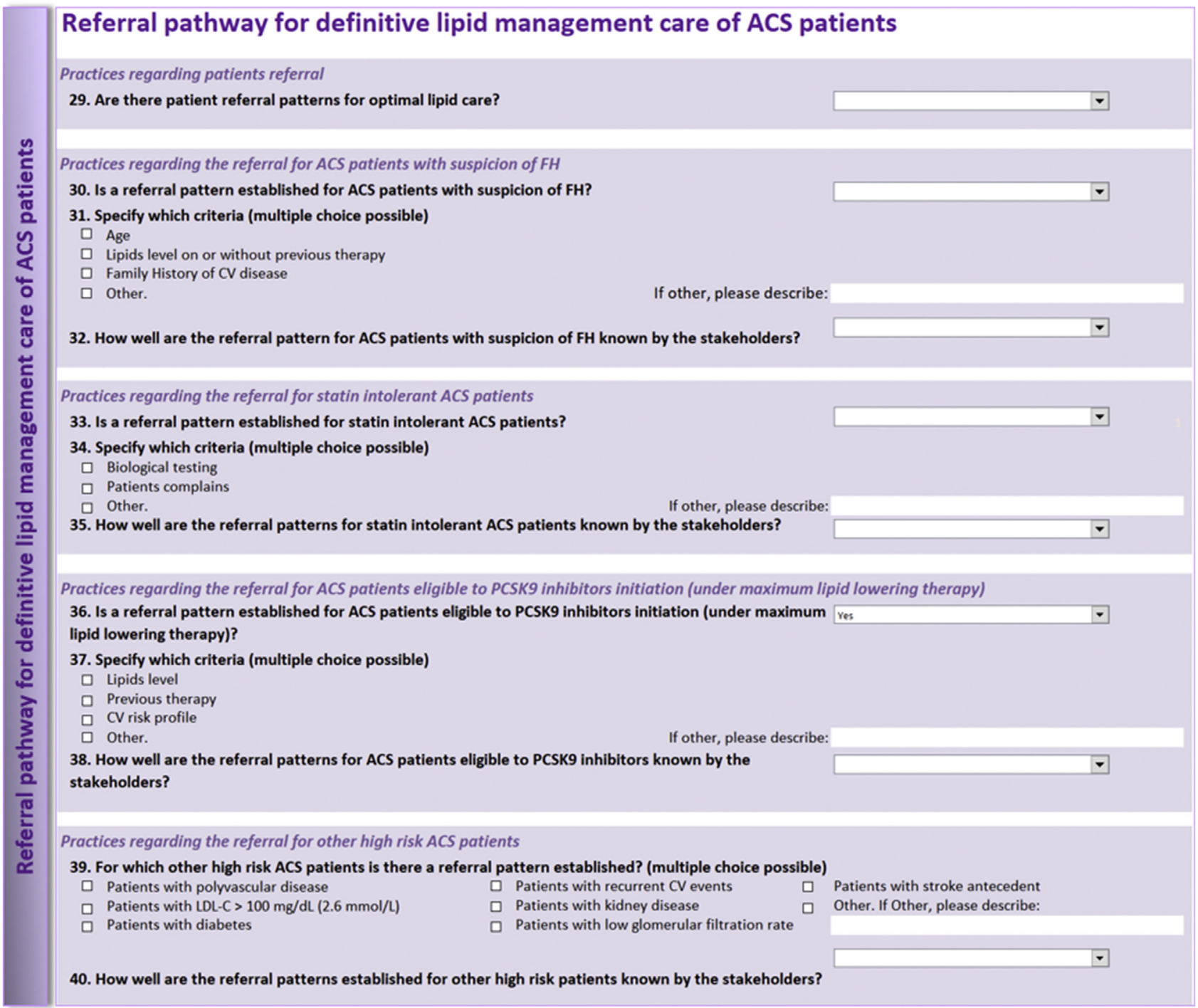

Fig. 4. Referral pathway for definitive lipid management of ACS patients.

overall survival following the acute event [6]. Of note, in the EuroPath I survey, only half of the patients had a rehabilitation/ secondary prevention program planned during follow-up [3]. In this section, physicians are required to insert information regarding the follow-up planning, including participation in a rehabilitation program, and the indication of the LDL-C goal. Furthermore, details on the indications included in the discharge letter must be stated (including the LDL-C goal, time to next LDL-C evaluation, the pharmacological approach to be adopted in case LDL-C goal is not reached, the timing for future follow-up visits) (Fig. 2). Finally, details on the information provided to the patient at discharge are also required, including indications on LDL-C goal, follow-up visit plan (including lipid profile monitoring), and lifestyle interventions (such as sport and diet) (Fig. 2).

The follow-up section includes information on lipid management practice at the time of the first follow-up visit and subsequent follow-ups (Fig. 3). This section focuses on the intensification of lipid-lowering therapy in those patients who did not achieve their

Fig. 5. Outcomes at 6 months to 1 year. 
LDL-C goal, and requires information on the clinical criteria considered for therapy intensification (such as lipid levels, individual CV risk profile, therapy tolerance) as well as the type of treatment intensification adopted (increase of statin dose, adding ezetimibe to the same statin dose or on top of the maximally tolerated dose, initiating a PSCK9 inhibitor). Furthermore, the timing of intensification of the therapy is required (Fig. 3).

The fourth section is focused on the referral pathway for lipid management of post-ACS patients, with special attention for patients suspected of $\mathrm{FH}$, statin-intolerant patients, patients eligible for a PCSK9 inhibitor, patients at high risk due to the presence of risk factors such as polyvascular disease, elevated LDL-C, diabetes, recurrent CV events, kidney disease or previous stroke (Fig. 4).

The last section requires information on the LDL-C levels achieved at 6 months to 1 year, and offers the opportunity to indicate which part of the local pathway should be refined or optimized, with the aim of further refining the application of the appropriate approach with these patients (Fig. 5).

\section{Report}

This second part of the tool is a brief report allowing to position the results obtained in each single section with respect to either the latest European guidelines or other European countries (as an example of current practice) (Fig. 6). This will allow the physician to define the capability of the local pathway for the lipid management of ACS patients to follow these high CV risk patients with the most appropriate approach and to achieve the lipid goal defined for their CV risk category.

\section{Next step}

The last section allows physicians to suggest actions to be implemented in order to further optimize the management of ACS

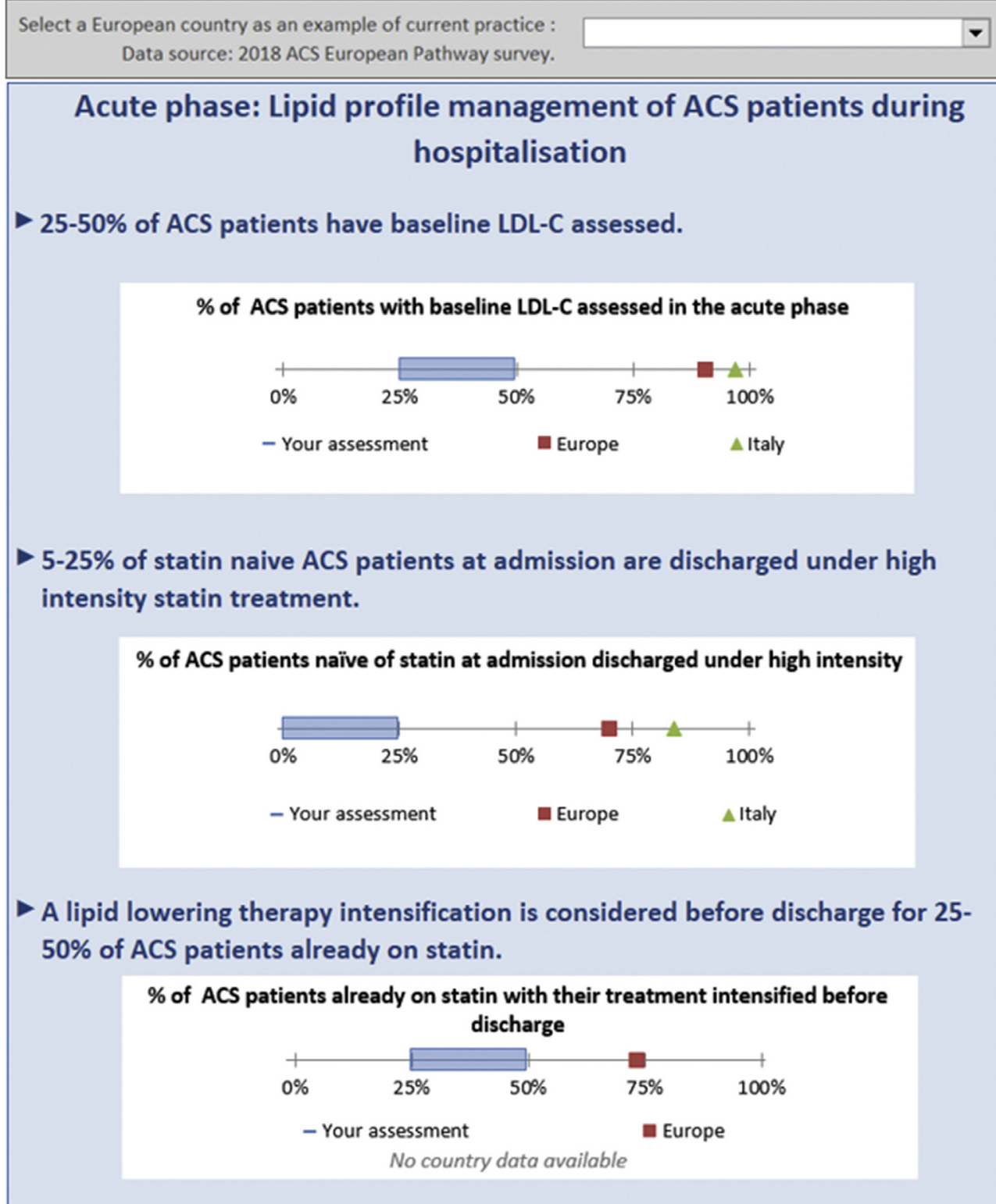

Fig. 6. Positioning of results with regards to other EU clinical practice stemming from the ACS Patient Pathway Project survey and the ESC/EAS Guidelines-An example. 
patients.

Evaluation of the ACS Lipid EuroPath tool by practicing physicians revealed that $80 \%$ found the tool useful, and $74 \%$ found the tool easy to use; the possibility to compare clinical practice to the ACS EuroPath survey and to the dyslipidaemias guidelines was judged as positive by $75 \%$.

\section{Discussion}

Post-ACS patients are at high risk of recurrent events and thus require a targeted pharmacological approach for optimal management of underlying risk factors. Hypercholesterolaemia represents a major risk factor in these patients, as several studies have shown that in clinical practice the achievement of the guidelines recommended LDL-C goal is obtained in a low percentage of patients (ref) $[3,7]$. This may be related to a broad range of factors; among them, the lack of common tools defining specific key steps for the management of patients with an ACS during the hospitalization (acute phase), at discharge, and at follow-up. The tool presented has been created with the aim to fill this gap, and to provide the physician with an instrument to optimize and improve lipid management in high CV risk patients. Furthermore, by comparing the results obtained by the physician with other examples of current practice would help define areas that can be potentially improved either at the individual or local level.

The development of this tool is based on the experience of previous studies. The ACS Patient Pathway Project (EuroPath I) survey was performed in 7 European countries with the aim of evaluating compliance to the recommendations from current ESC/ EAS guidelines for the management of ACS patients and the effectiveness of secondary prevention in this high CV risk population [3]. The results showed that lipid management is still suboptimal despite the availability of highly effective lipid-lowering drugs, which exposes these patients to high risk of recurrent events. On the other hand, European initiatives in which local algorithms specific for ACS patients have been routinely applied successfully, translated into a significantly higher achievement of LDL-C goals due to the presence of lipid teams to which patients are referred to after discharge and the wide use of high intensity lipid-lowering therapies [4]. For these reasons, attention is drawn to the continuous evaluation of the lipid profile and lipid-lowering therapies at all stages (acute phase, discharge, and follow-up), as the benefits of statins in secondary prevention have been unequivocally demonstrated, and a large number of trials have shown the benefits of early and intensive statin therapy in ACS [6]. The relevance of the lipid-lowering approach in ACS patients has also been reviewed by the most recent ESC/EAS guidelines in which the addition of a PCSK9 inhibitor to current therapy is recommended when the LDL$C$ goal is not achieved after 4-6 weeks despite maximal tolerated statin therapy and ezetimibe [5].

Furthermore, there is emphasis on the importance of the role of screening for FH in ACS patients. In fact, although it is known that this inherited condition is common in the general population $(\sim 1: 250)$ [8] and is even more common among ACS patients [9-13], several studies provided evidence that FH screening within ACS patient population remains largely unapplied. FH confers by itself a high- or very-high CV risk, and, due to the lifelong exposure to elevated levels of LDL-C, the occurrence of cardiovascular events at an early age compared with the general population must alert the cardiologist to start a specific pathway for the correct diagnosis and the appropriate pharmacological approach.

The intention is that application of this simple self-assessment tool will help bridge the guidelines to practice gap evident in the optimization of lipid management in ACS patients. To access the tool, please visit the page www.sitecs.it.

\section{CRediT authorship contribution statement}

Alessandro Sionis: Conceptualization, Methodology, Investigation, Supervision. Alberico L. Catapano: Conceptualization, Methodology, Investigation, Supervision. Gaetano M. De Ferrari: Conceptualization, Supervision, Methodology, Investigation. Dariusz Dudek: Conceptualization, Supervision, Methodology, Investigation. J. Wouter Jukema: Conceptualization, Supervision, Methodology, Investigation. Angela Pirillo: Writing - Original Draft, Writing - Review \& Editing, Visualization François Schiele: Conceptualization, Supervision, Methodology, Investigation. Azfar Zaman: Conceptualization, Supervision, Methodology, Investigation. Jose L. Zamorano: Conceptualization, Supervision, Methodology, Investigation.

\section{Declaration of competing interest}

AS or his department have received research grants and/or speaker honoraria from Amgen, Astra-Zeneca, Dalcor, DaichiiSankyo, Esperion, Ferrer, Novartis, Orion-Pharma, Pfizer, Sanofi Aventis, Servier, Sphingotech, Singulex and Zoll; additionally from the following agencies: Sociedad Española de Cardiología; Instituto de Salud Carlos III (ISCIII). ALC reports grants from Amgen, Sanofi, Regeneron personal fees from Merck, Sanofi, Regeneron, AstraZeneca, Amgen, Novartis, outside the submitted work. GDF has received research grant support from Amgen, Merck, and Novartis; has served as a consultant for Amgen, Boston Scientific, Livanova, Merck, and Sigma-Tau; and received lecturing fees from Amgen, Merck, and Sigma-Tau. DD has nothing to disclose. JWJ/his department has received research grants from and/or was speaker (with or without lecture fees) on a.o.(CME accredited) meetings sponsored by Amgen, Athera, Astra-Zeneca, Biotronik, Boston Scientific, Dalcor, Daiichi Sankyo, Lilly, Medtronic, Merck-ScheringPlough, Pfizer, Roche, Sanofi Aventis, The Medicine Company, the Netherlands Heart Foundation, CardioVascular Research the Netherlands (CVON), the Netherlands Heart Institute and the European Community Framework KP7 Programme. UL reports grant support from Bayer and Novartis, and lecture honoraria from AstraZeneca, Amgen, Bayer, Sanofi, Berlin Chemie, Novartis, Abbott, and the Medicines Company. AP has nothing to disclose. FS has received research grants and/or speaker honoraria from: Amgen, Astra-Zeneca, Bayer, Dalcor, Lilly, Novonordisk, MSD, Mylan, Novartis, Sanofi Aventis, Servier and Terumo. AZ has received consulting fees from Miracor. JLZ reports research grants from Abbott and speaker honoraria from Bayer, Pfizer, and Daichii.

\section{Acknowledgments}

The presentation of data contained in this work at the annual meeting of SITeCS (Società Italiana di Terapia Clinica e Sperimentale) was realized with the institutional support of Sanofi. The sponsor of this activity had no role in the design, interpretation, decision to publish, or writing the manuscript. The work of ALC has been supported by Ministry of Health - Ricerca Corrente - IRCCS MultiMedica, PRIN 2017H5F943 and ERANET ER-2017-2364981. This article is part of a Supplement entitled "Plasma lipids and cardiovascular risk: Nutritional and therapeutic approaches" published with support from Società Italiana di Terapia Clinica e Sperimentale (SITeCS).

\section{References}

[1] Jernberg T, Hasvold P, Henriksson M, et al. Cardiovascular risk in postmyocardial infarction patients: nationwide real world data demonstrate the importance of a long-term perspective. Eur Heart J 2015;36:1163-70. 
[2] Fox KA, Carruthers KF, Dunbar DR, et al. Underestimated and underrecognized: the late consequences of acute coronary syndrome (GRACE UKBelgian Study). Eur Heart J 2010;31:2755-64.

[3] Landmesser, U, Pirillo, A, Farnier, M, et al., Lipid-lowering therapy and lowdensity lipoprotein cholesterol goal achievement in patients with acute coronary syndromes: the ACS Patient Pathway Project submitted for publication.

[4] Alings, M, Descamps, O, Guillon, B, et al., Implementation of clinical practices and pathways optimising ACS patients lipid management: focus on eight European initiatives submitted for publication.

[5] Mach F, Baigent C, Catapano AL, et al. ESC/EAS Guidelines for the management of dyslipidaemias: lipid modification to reduce cardiovascular risk. Eur Heart J 2019;41:111-88.

[6] Ibanez B, James S, Agewall S, et al. ESC Guidelines for the management of acute myocardial infarction in patients presenting with ST-segment elevation: the Task Force for the management of acute myocardial infarction in patients presenting with ST-segment elevation of the European Society of Cardiology (ESC). Eur Heart J 2017;39:119-77.

[7] De Backer G, Jankowski P, Kotseva K, et al. Management of dyslipidaemia in patients with coronary heart disease: results from the ESC-EORP EUROASPIRE V survey in 27 countries. Atherosclerosis 2019;285:135-46.

[8] Nordestgaard BG, Chapman MJ, Humphries SE, et al. Familial hypercholesterolaemia is underdiagnosed and undertreated in the general population: guidance for clinicians to prevent coronary heart disease: consensus statement of the European Atherosclerosis Society. Eur Heart J 2013;34:3478-3490a.

[9] De Backer G, Besseling J, Chapman J, et al. Prevalence and management of familial hypercholesterolaemia in coronary patients: an analysis of EUROASPIRE IV, a study of the European Society of Cardiology. Atherosclerosis 2015;241:169-75.

[10] Pang J, Poulter EB, Bell DA, et al. Frequency of familial hypercholesterolemia in patients with early-onset coronary artery disease admitted to a coronary care unit. J Clin Lipidol 2015;9:703-8.

[11] Nanchen D, Gencer B, Auer R, et al. Prevalence and management of familial hypercholesterolaemia in patients with acute coronary syndromes. Eur Heart J 2015;36:2438-45.

[12] Faggiano P, Pirillo A, Griffo R, et al. Prevalence and management of familial hypercholesterolemia in patients with coronary artery disease: the heredity survey. Int J Cardiol 2018;252:193-8.

[13] Kramer AI, Trinder M, Brunham LR. Estimating the prevalence of familial hypercholesterolemia in acute coronary syndrome: a systematic review and meta-analysis. Can J Cardiol 2019;35:1322-31. 